\section{Research Square}

Preprints are preliminary reports that have not undergone peer review.

They should not be considered conclusive, used to inform clinical practice, or referenced by the media as validated information.

\title{
The Role of Personality Associated with In-Hospital Delay in Intravenous Thrombolysis for Acute Ischemic Stroke
}

\author{
Zhenchan Lu \\ Huzhou central hospital \\ Caixia Qiu \\ Huzhou central hospital \\ Xiangyan Yang \\ Huzhou central hospital \\ Honggang $\mathrm{Ma}$ \\ Huzhou central hospital \\ Shuang Shen \\ Huzhou central hospital \\ Shenlin Xia ( 13735140036@163.com ) \\ Huzhou central hospital
}

\section{Original research}

Keywords: Big Five Personality Traits, Physicians, In-Hospital Delay, Ischemic Stroke, Intravenous Thrombolysis

Posted Date: November 13th, 2020

DOI: https://doi.org/10.21203/rs.3.rs-104374/v1

License: (9) This work is licensed under a Creative Commons Attribution 4.0 International License. Read Full License 


\section{Abstract}

Background: Decreasing the in-hospital delay is one of the critical effective strategies for thrombolytic therapy for acute ischemic stroke. we examined whether physician personality traits are associated with in-hospital delay in conducting treatment of intravenous thrombolysis for acute ischemic stroke among neurologists.

Methods: Overall 354 consecutive patients who received intravenous thrombolysis during a 2.5 year-period in emergency department were included. Self-reported questionnaires of the Big Five Inventory and demographic characteristics were distributed among 13 neurologists. Multivariable analysis was performed to explore the effects of the Big Five Personality Traits on in-hospital delay for acute ischemic stroke.

Results: The traits of agreeableness in all physicians decreased the likelihood of in-hospital delay (OR: 0.831, 95\% Cl: 0.766-0.901, $\mathrm{p}<0.001)$. The traits of openness in female physicians (OR:0.646; $95 \% \mathrm{Cl}: 0.469-0.890 ; p=0.008)$ and the traits of extraversion in male physicians (OR:0.613; $95 \% \mathrm{Cl}: 0.475-0.791 ; \mathrm{p}<0.001$ ) decreased the likelihood of in-hospital delay. The traits of conscientiousness in female (OR: 1.713, 95\% Cl: 1.209-2.427; $p=0.002)$ and the traits of openness in male (OR: 1.431; 95\% Cl: 1.802-1.892; $p=0.012)$ increased the likelihood of in-hospital delay.

Conclusions: The study demonstrate that the personality traits of physician are associated with in-hospital delay for thrombolytic therapy in acute ischemic stroke.

\section{Introduction}

According to the Chinese and international guidelines regarding acute ischemic stroke (AIS), intravenous thrombolysis (IVT, i.e. recombinant tissue plasminogen activator (tPA) and urokinase) should be given within 4.5 hours after the onset of the symptoms in the treatment of $\mathrm{AIS}^{[1-2]}$. The benefits of reperfusion therapy are highly time-dependent, with the earlier the IVT is applied, the better the outcome of the treatment of $\mathrm{AIS}^{[3]}$. The door-to-needle time (DNT), the sign of in-hospital delay or not, guideline-recommended, the time from arrival at hospital to time of IVT, constitute the fundamental indicators of the stroke management in the acute phase ${ }^{[4]}$. Previous studies have developed and demonstrated critical effective strategies for shortening the DNT, including pre-notification, activation of the stroke team, continuously optimizing the systematic protocol (registration process, computed tomography, administration of alteplase in the scanner etc. $)^{[5]}$, and performing the Get With the Guidelines (GWTG)-Stroke program ${ }^{[6]}$.

Despite these major developments, situational factors influencing the shortening of the DNT, as well as the individual personality factors influencing the time of intravenous thrombolysis remain unknown. Personality refers to the distinctive patterns of thinking, feeling, and behavior of an individual[ ${ }^{[7]}$. Of note, personality can be categorized into five broad traits, namely $\mathrm{C}$ (Conscientiousness: efficient, organized, planning, reliable, responsible, thorough, disciplined, careful), A (agreeableness: appreciative, forgiving, generous, kind, sympathetic, trusting, helpful), $\mathrm{N}$ (Neuroticism: anxious, self-pitying, tense, touchy, unstable, worrying, pessimistic), $\mathrm{O}$ (Openness: artistic, curious, imaginative, insightful, original, wide interests openness, spontaneous), and E (extraversion: active, assertive, energetic, enthusiastic, outgoing, talkative, sociable, fun-loving). Together, these traits make up the five-factor model (the acronym CANOE) of personality, also known as the Big Five Model ${ }^{[8-9]}$. A preliminary study into the personalities of neurologists using the TenItem Personality Inventory (TIPI), an internationally validated measure of the five-factor model of personality dimensions ${ }^{[10]}$, found higher conscientiousness in neurologists ${ }^{[11]}$. Whether these physician traits are related to the medical practice aspects of each individual physician in administering the thrombolytic agent to the patient remains unclear. Therefore, in the present study, we hypothesized that there is a relationship between the Five Factor Model of physician personality and DNT (in-hospital delay) in conducting treatment of intravenous thrombolysis for AIS among neurologists.

\section{Methods}

\section{Subjects}

This was a retrospective survey based on the data obtained from the Comprehensive Stroke Center of Huzhou Central Hospital, which was officially established in Jan 2017. All patients diagnosed with stroke were given specialty treatment by neurologists in the emergency department (ED). Therefore, the data for this study was collected from Jan 2018 to Jun 2020. The study consists of two 
subjects (neurologists and patients). Overall, 13 neurologists worked in shifts in the neurological ED. All these neurologists completed a Big Five Inventory to assess their personality traits. This inventory was originally developed by John et a ${ }^{[8]}$.and was provided in a Chinese translation, Chinese Big Five Personality Inventory Brief Version (CBF-PI-B), by Wang et al ${ }^{[12]}$. The neurologists additionally completed a questionnaire seeking information on gender, ethnicity, educational background, and medical experience based on the years of the acquired clinical neurology experience. The percentage proportion of the female subjects was $46 \%$ [ $n=6$; mean age 35.5 years (SD 3.5), range 30-39] and that of male was $54 \%$ [ $n=7$; mean age 41.4 years (SD3.7), range 38-47]. All the 13 neurologists completed the list between Jun and July 2020. Table 1 shows the details of every neurologist's information.

Table 1

The baseline characteristics of the 13 neurologists

\begin{tabular}{|c|c|c|c|c|c|c|}
\hline Num & Gender & Age (Years) & Ethnic & Education & Experience (Years) & DNT $(\min )^{*}$ \\
\hline 1 & male & 47 & Han & master & 15 & $52(41-71)$ \\
\hline 2 & male & 45 & Han & bachelor & 16 & $40(33-47)$ \\
\hline 3 & male & 42 & Han & master & 16 & $50(35-67)$ \\
\hline 4 & male & 40 & Han & bachelor & 12 & $50(43-61)$ \\
\hline 5 & female & 39 & Han & bachelor & 12 & $60(43-66)$ \\
\hline 6 & female & 38 & Han & master & 11 & $51(41-60)$ \\
\hline 7 & female & 38 & Han & bachelor & 11 & $45(36-65)$ \\
\hline 8 & male & 38 & Han & master & 11 & $40.5(35-49)$ \\
\hline 9 & male & 38 & Han & master & 10 & $36(31-43)$ \\
\hline 10 & male & 38 & Han & master & 8 & $47(35-54)$ \\
\hline 11 & female & 35 & Han & bachelor & 6 & $43(39-46)$ \\
\hline 12 & female & 33 & Han & master & 5 & $62(46-66)$ \\
\hline 13 & female & 30 & Han & master & 3 & $66(58-74)$ \\
\hline
\end{tabular}

All adult ( $\geq 18$ years) patients admitted from Jan 2018 to Jun 2020 to the stroke center with an acute ischemic stroke receiving IVT were included. Patients who were transferred from the primary stroke unit to receive the direct procedure of IVT and patients with inhospital stroke, incomplete or contradictory documentation, and stroke mimic were excluded. The Human Subjects Review Committee of the Huzhou Central Hospital approved this research. All questionnaires were administered to those who provided informed consent.

\section{Instruments}

The CBF-PI-B administered as a self-report measure consisting of 40 items, easy to understand questions and dovetail with Chinese idiomatic expressions, was used to evaluate neurologist personality traits (five personality dimensions). Items $5,8,13,15,18,32$, and 36 were scored reversely. Each item was answered on a six-point, Likert-type scale, ranging from 1 ("disagree strongly") to 6 ("agree strongly"). The Score of each item was summarized separately.

\section{Time Parameters And Other Data}

The main observation times were measured in minutes. Door-to-needle time (DNT), according to the Campaign Manual ${ }^{[13]}$, in-hospital Delayed treatment was defined as a DNT > 45 min. Onset-to-door time (ODT), door-to-imaging time (DTI), door-to-laboratory time (DTL), and final-test-to-needle time (FTN), the time interval between the time of obtaining the result of the last screening test and the needle time)) were recorded in minutes. The stroke time form was a standard pre-designed structured time form and it was filled by 
an independent trained paramedic, under supervision of an emergency physician. The data for the times was obtained: time of stroke onset (the time the patient or others first noted the abnormal symptoms), arrival at the stroke center, evaluation of head computer tomography, laboratory results, communication and signing the informed consent agreement and the bolus administration of thrombolytic drugs (tPA or urokinase). In addition to the above-mentioned time points, we observed demographics, blood pressure, risk factors of stroke, previous history of stroke, use of antithrombotic medications, electrocardiogram, and the National Institute of Health Stroke Scale (NIHSS) scores.

\section{Statistical Analysis}

Data are expressed as mean \pm Sd. Deviation (SD), median (interquartile range [IQR]), or percentage, as appropriate. Univariate analyses (Mann-Whitney $U$ test for continuous variables, and $\chi^{2}$ test for categorical variables, as appropriate) were performed to assess the association of each of the independent variables and dependent variable. Pearson's correlation analysis was used to determine whether these factors were likely to affect the correlation. Logistic regression models evaluated the association between the levels of five personality dimensions and in-hospital delay. All the statistical analyses were performed using SPSS v22.0 (IBM Corp. Released 2013. IBM SPSS Statistics for Windows, Version 22.0. Armonk, NY: IBM Corp)]. All $p$ values were 2-tailed, and the differences were considered significant at $\mathrm{p}<0.05$.

\section{Results}

During the 30-month study period, all of the 414 stroke cases with ischemic stroke were given IVT in the ED in the study hospital. Among the 414 stroke patients, overall 60 patients were excluded: 49 patients because they were transferred from the primary stroke unit, 5 patients because they were in-hospital stroke cases, 3 patients as they were stroke mimic cases (benzodiazepine poisoning, Datura poisoning, aortic dissection), and 3 patients with incomplete or contradictory data. Finally, 354 ischemic stroke patients were included in the study as shown in Fig. 1.

There was no significant change in the trend of DNTs (M (IQR), M, median, IQR, interquartile range), Jan to Dec 2018 (118cases 46 (38-62)), Jan to Dec 2019 (148cases 47 (38-60)), Jan to Jun 2020 (88 cases 44 (35-54)), during the study period of 30 months, (P $=0.324)$ as shown in Fig. 2 .

Although the mean scores for the CBF-PI-B were not significantly different across all the dimensions, gender heterogeneity was reported in the $\mathrm{N}$ (neuroticism) and $\mathrm{O}$ (openness) traits in all the 13 neurologists (Fig. 3).

Among the 354 patients, the median door-to-needle time score for IVS was 46 [IQR 37-60] min. Of which, 171 patients (48.3\%) were classified in the effective treatment group (DNT $\leqq 45$ min median DNT score: 37 [IQR 33-42] min) and 183 patients (51.7\%) were classified in the delayed treatment group (DNT > 45 min median DNT score: 60 [IQR 51-70] min). Compared with the delayed treatment group, the time of DTI, DTL, FTN, and DNT were remarkably shortened in the effective treatment group $($ all $p<0.01)$ (Table 2). 
Table 2

Patient and physician characteristics according to effective treatment (DNT $\leqq 45 \mathrm{~min}$ ) or not

\begin{tabular}{|c|c|c|c|}
\hline & $\begin{array}{l}\text { Effective treatment } \\
(n=171,48.3 \%)\end{array}$ & $\begin{array}{l}\text { Delayed treatment } \\
(n=183,51.7 \%)\end{array}$ & $P$ value \\
\hline \multicolumn{4}{|l|}{ Patient characteristics } \\
\hline Age in years, mean $\pm S D$ & $71.0 \pm 12.1$ & $70.8 \pm 12.9$ & 0.876 \\
\hline Gender, Female, No. (\%) & $66(38.6)$ & $88(48.1)$ & 0.072 \\
\hline Risk factors, No. (\%) & $129(75.4)$ & $140(76.5)$ & 0.815 \\
\hline Hypertension & $36(21.1)$ & $42(22.9)$ & 0.667 \\
\hline Diabetes mellitus & $32(18.7)$ & $34(18.6)$ & 0.974 \\
\hline Dyslipidemia & $44(25.7)$ & $45(24.5)$ & 0.804 \\
\hline Atrial fibrillation & $39(22.8)$ & $36(19.7)$ & 0.476 \\
\hline Alcohol consumption & $53(30.9)$ & $55(30.1)$ & 0.847 \\
\hline Current smoker & $50(29.2)$ & $52(28.4)$ & 0.864 \\
\hline Hyperhomocysteinemia & $166(97.1)$ & $174(95.1)$ & 0.418 \\
\hline Clear onset, No. (\%) & $8(4.7)$ & $6(3.3)$ & 0.590 \\
\hline Previous stroke, №. (\%) & $14(8.2)$ & $16(8.7)$ & 0.851 \\
\hline On antiplatelet drugs at admission, No. (\%) & $99(57.9)$ & $96(52.4)$ & 0.232 \\
\hline Ambulance, №. (\%)* & $106(69-161)$ & $115(65-150)$ & 0.972 \\
\hline ODT in mins, median (IQR) & $20(15-26)$ & $29(22-36)$ & $<0.001$ \\
\hline DTL in mins, median (IQR) & $15(11-19)$ & $23(16-28)$ & $<0.001$ \\
\hline DTI in mins, median (IQR) & $15(10-21)$ & $28(18-40)$ & $<0.001$ \\
\hline FTN in mins, median (IQR) & $37(33-42)$ & $60(51-70)$ & $<0.001$ \\
\hline DNT in mins, median (IQR) & $10.9 \pm 8.5$ & $10.8 \pm 8.2$ & 0.252 \\
\hline $\mathrm{NIHSS}$, mean \pm SD & & & \\
\hline
\end{tabular}

* Patients arriving by ambulance to the emergency department, the years of the acquired clinical neurology experience. IQR: interquartile range, NIHSS: National Institute of Health Stroke Scale. DNT: door-to-needle time, ODT: onset-to-door time, DTI: doorto-imaging time, DTL: door-to-laboratory time and FTN: final-test-to-needle time, C (Conscientiousness), A (Agreeableness), N (Neuroticism), O (Openness) and E (extraversion). 


\begin{tabular}{|c|c|c|c|}
\hline & $\begin{array}{l}\text { Effective treatment } \\
(n=171,48.3 \%)\end{array}$ & $\begin{array}{l}\text { Delayed treatment } \\
(n=183,51.7 \%)\end{array}$ & $P$ value \\
\hline Age in years, mean $\pm S D$ & $39.6 \pm 3.5$ & $38.7 \pm 4.3$ & 0.417 \\
\hline Gender, Female, No. (\%) & $39(22.8)$ & $74(40.4)$ & $<0.001$ \\
\hline Experience in years, mean $\pm S D \#$ & $10.9 \pm 8.5$ & $10.8 \pm 8.2$ & 0.362 \\
\hline C. mean \pm SD & $31.9 \pm 6.2$ & $32.3 \pm 5.9$ & 0.011 \\
\hline A. mean $\pm S D$ & $37.9 \pm 7.4$ & $37.4 \pm 6.8$ & 0.921 \\
\hline N. mean $\pm S D$ & $20.0 \pm 5.5$ & $21.4 \pm 5.2$ & 0.040 \\
\hline O. mean \pm SD & $31.0 \pm 9.3$ & $28.7 \pm 8.5$ & 0.011 \\
\hline E. mean $\pm S D$ & $28.9 \pm 3.3$ & $27.0 \pm 4.2$ & $<0.001$ \\
\hline
\end{tabular}

Meanwhile, our analysis revealed significant but small size differences among groups for the four personality factors (conscientiousness, neuroticism, openness, and extraversion) (all $p<0.05)$. Compared with the delayed treatment group, lower scores of conscientiousness and neuroticism, and higher scores of openness and extraversion were observed in the effective treatment group $(\mathrm{p}<0.05)($ Table 2$)$.

There was a moderate correlation between extraversion and DNT $(r=-0.314, p<0.001)$ among the all physicians. However, there was a weak correlation with regards to gender: female $(r=-0.290, p<0.001)$, male $(r=-0.222, p<0.001)$, respectively. Neuroticism was strongly positively associated with DNT $(r=0.360, p<0.001)$ in the female physicians, but was not statistically significant in male physicians. Weak positive correlations were also found between openness and DNT in the male, as well as among all the physicians. The correlations between the five personality traits and DNT are shown in Fig. 4.

Further multivariable analysis indicated that the traits of neuroticism (OR: $0.925 ; 95 \% \mathrm{Cl}: 0.863-0.991 ; \mathrm{p}=0.027$ ) and extraversion (OR: $0.831,95 \% \mathrm{Cl}: 0.766-0.901, \mathrm{p}<0.001$ ) were negatively associated with delayed treatment. However, the conscientiousness traits were positively associated with delayed treatment (DNT > $45 \mathrm{~min}$ ) among all the physicians (OR: 1.116, 95\% Cl: 1.057-1.178; $p<$ 0.001). Gender differences were also found in the relationship between personality traits and delayed treatment. The traits of agreeableness (OR: 0.571; $95 \% \mathrm{Cl}: 0.395-0.825 ; \mathrm{p}=0.003$ ), openness (OR: $0.646 ; 95 \% \mathrm{Cl}: 0.469-0.890 ; \mathrm{p}=0.008)$ in female neurologists and the traits of agreeableness (OR: $0.705 ; 95 \% \mathrm{Cl}: 0.539-0.922 ; \mathrm{p}=0.011)$, extraversion (OR: $0.613 ; 95 \%$ Cl: $0.475-$ $0.791 ; p<0.001)$ in male neurologists decreased the likelihood of delayed treatment. The traits of conscientiousness in female physicians (OR: $1.713 ; 95 \% \mathrm{Cl}: 1.209-2.427, \mathrm{p}=0.002)$ and the traits of openness in male physicians (OR: $1.431 ; 95 \%$ Cl: $1.802-$ 1.892; $p=0.012)$ increased the likelihood of delayed treatment. However, the acquired clinical neurology experience decrease the likelihood of delayed treatment irrespective of the gender differences (OR: 0.894; 95\% Cl: 0.822-0.972; p =0.009) (Table 3). 
Table 3

Multivariate logistic regression analysis of factors (including Big Five Dimensions) related to delayed treatment (DNT > 45 min) for stroke

\begin{tabular}{|c|c|c|c|c|c|c|c|c|c|c|c|c|}
\hline \multirow[t]{2}{*}{ Variations } & \multicolumn{4}{|l|}{ Total } & \multicolumn{4}{|c|}{ Female } & \multicolumn{4}{|l|}{ Male } \\
\hline & $\beta$ & SE & $\begin{array}{l}\text { OR } \\
(95 \% \\
\text { Cl) }\end{array}$ & $\mathbf{P}$ & $\beta$ & SE & $\begin{array}{l}\text { OR } \\
(95 \% \\
\text { Cl) }\end{array}$ & $\mathbf{P}$ & $\beta$ & SE & $\begin{array}{l}\text { OR } \\
(95 \% \\
\text { Cl) }\end{array}$ & $\mathbf{P}$ \\
\hline $\mathrm{C}$ & 0.110 & 0.027 & $\begin{array}{l}1.116 \\
(1.057- \\
1.178)\end{array}$ & $\begin{array}{l}< \\
0.001\end{array}$ & 0.538 & 0.178 & $\begin{array}{l}1.713 \\
(1.209- \\
2.427)\end{array}$ & 0.002 & 0.070 & 0.039 & $\begin{array}{l}1.073 \\
(0.994- \\
1.158)\end{array}$ & 0.071 \\
\hline$A$ & -0.052 & 0.030 & $\begin{array}{l}0.950 \\
(0.896- \\
1.006)\end{array}$ & 0.081 & -0.560 & 0.188 & $\begin{array}{l}0.571 \\
(0.395- \\
0.825)\end{array}$ & 0.003 & -0.349 & 0.137 & $\begin{array}{l}0.705 \\
(0.539- \\
0.922)\end{array}$ & 0.011 \\
\hline $\mathrm{N}$ & -0.078 & 0.035 & $\begin{array}{l}0.925 \\
(0.863- \\
0.991)\end{array}$ & 0.027 & & & & n.s. & & & & n.s. \\
\hline 0 & & & & n.s. & -0.437 & 0.164 & $\begin{array}{l}0.646 \\
(0.469- \\
0.890)\end{array}$ & 0.008 & 0.358 & 0.143 & $\begin{array}{l}1.431 \\
(1.802- \\
1.892)\end{array}$ & 0.012 \\
\hline$E$ & -0.186 & 0.041 & $\begin{array}{l}0.831 \\
(0.766- \\
0.901)\end{array}$ & $\begin{array}{l}< \\
0.001\end{array}$ & & & & n.s. & -0.489 & 0.130 & $\begin{array}{l}0.613 \\
(0.475- \\
0.791)\end{array}$ & $\begin{array}{l}< \\
0.001\end{array}$ \\
\hline Experience* & -0.112 & 0.043 & $\begin{array}{l}0.894 \\
(0.822- \\
0.972)\end{array}$ & 0.009 & -0.533 & 0.201 & $\begin{array}{l}0.587 \\
(0.395- \\
0.870)\end{array}$ & 0.008 & -0.340 & 0.120 & $\begin{array}{l}0.712 \\
(0.563- \\
0.900)\end{array}$ & 0.005 \\
\hline
\end{tabular}

\section{Discussion}

This is the first study on the association between physician personality traits and the in-hospital delay for stroke thrombolysis treatment in China. In this study, we demonstrated that certain personality traits among physicians are associated with door-to-needle time for thrombolytic therapy in acute ischemic stroke. The extraversion trait shortened the door-to-needle time. However, the trait of conscientiousness, to some extent, postponed the time of procedure for thrombolysis treatment.

"Time is Brain, Every Minute Counts" has been widely acknowledged, with every minute the thrombolytic treatment for stroke is started earlier, the higher the odds of a good outcome ${ }^{[14-15]}$. The importance of constant improvement of in-hospital treatment routines cannot be overemphasized. In addition, the door-to-needle time is a critical factor in achieving prospective outcomes after an ischemic stroke ${ }^{[15]}$. Nevertheless, there is a wide chasm in access to essential and emergency treatment between high and low/middle income countries ${ }^{[4,16-17]}$.

In China, the government instituted ambitious strategies for the prevention and treatment of stroke ten years ago ${ }^{[18]}$. Owing to the support of the Stroke Prevention Project Committee (founded in 2011), affiliated to the National Health Commission, the Stroke Center of Huzhou Central Hospital was officially established in Jan 2017. Moreover, the Acute Stroke Care Map was implemented in Oct 2018 in Huzhou district (Zhejiang Province, East China). With this, the improvement in the Stroke Center of Huzhou Central Hospital is noticeable, with the percentage of stroke patients with acute ischemic stroke (within 6hrs) receiving intravenous thrombolysis improving from less than 1\% in 2013 to up to 8\% in 2019, and the median door-to-needle time shortened from 85.1 min in 2013 to $47.2 \mathrm{~min}$ in 2019 for stroke thrombolysis treatment. It is obvious that there is improvement in the rapid administration of intravenous thrombolysis and the reduction of door-to-needle time in China ${ }^{[1,18]}$, The results of this study demonstrated that the prolonged DTI, DTL, and FTN accounted for the in-hospital delay. Our results are consistent with the findings of a previous study, which documented that FTN contributed significantly to in-hospital delay ${ }^{[19]}$. FTN constitutes the time involved in the process of decision-making for IVT. The decision-making process is composed of the decision of the family of the patient and the advice of the 
physician. Moreover, considering the tense between the doctor-patient relationships in China ${ }^{[20]}$, the simple decision making process becomes extremely challenging.

In our study, we found that higher levels of extraversion were associated with a lower proportion of in-hospital delay. Physicians during an emergency may require more enthusiasm, activeness, and energy in dealing with critical situations. Our results are consistent with the findings of a previous study conducted in Germany, which demonstrated that the "resilient crisis manager" personality characteristics as suitable for emergency medical personnel ${ }^{[21]}$. Our subgroup analysis with respect to gender indicated that the personality traits of extraversion in male neurologists were associated with a lower likelihood of in-hospital delay. However, the advantage of extraversion was not reported in female physicians. This could be because Chinese female physicians are more reserved, introverted, fond of tranquility, overly considerate, and habituated to self-restraint ${ }^{[22]}$.

Moreover, our study showed that conscientiousness was associated with moderate in-hospital delay, especially in female physicians and the correlation decreased in male physicians. The personality traits of conscientiousness include organization, planning, reliable, responsible, and thorough. High levels of conscientiousness have been reported as desirable traits in a previous study among neurologists and neurosurgeons ${ }^{[11]}$. This medical professionalism among physicians may transform into a weakness in the surrounding when racing against time. Some physicians may take time performing head magnetic resonance imaging for accurate diagnosis in patients with a minor stroke. In addition, they take time weighing each patient in the emergency department, while others consider that it should be done only when patients do not know their weight. Of note, it takes $3-5$ minutes to take the weight on a self-made weighing scale (Fig. 5).

We also found that the agreeableness personality decreased the in-hospital delay for stroke thrombolysis. The appreciative, forgiving, kind, and sympathetic traits were shown to play a positive role in communicating with patients and their family members. Successful communication forms the basis of the final decision-making process for thrombolytic therapy. This could be attributed to empathy, which is an essential element in medical practice ${ }^{[23]}$. Previously, agreeableness has been demonstrated to be relatively associated with empathy in both Chinese and Japanese medical students ${ }^{[24-25]}$.

In this study, we established that neuroticism and openness personality traits have paradoxical effects on in-hospital delay. In the rough correlation analysis, the higher the scores of neuroticism, the more likely the in-hospital delay, especially among female physicians. However, after multivariate regression analysis, corrected factors, such as clinical neurology experience, age, etc., indicated that neuroticism could be an effective protective factor for effective treatment. Previous studies have suggested an important link between perfectionism and neuroticism ${ }^{[26]}$, and that neuroticism is correlated with the extent of anxiety ${ }^{[27]}$, which might be more conducive to emergencies to a certain extent. Nevertheless, it should also be noted that neuroticism is prone to burnout syndrome ${ }^{[28]}$. On the other hand, we showed that the openness scores were lower among female physicians than male physicians. Therefore, the traits of curiosity could help improve work efficiency in the process of thrombolysis among the female physicians. With regards to the male physicians, because of the artistic, original and wide interest traits concerning openness, they are more flexible in the real-world thrombolysis treatment for stroke. For instance, when the patient and his/her family decline to receive thrombolysis therapy due to financial limitations, the physician with more openness would recommend the alternative medicine of urokinase. Urokinase is one-tenth the price of Alteplase in China. Although this medical behavior may increase the DNT of thrombolysis, they would increase the percentage of thrombolysis treatments, and attain reperfusion opportunity for patients.

Moreover, we established a negative correlation between the acquired clinical neurology experiences and the in-hospital delay, regardless of the big five personality traits. With the accumulation of clinical experience, some of the personality disadvantages might get weak, consistent with the findings of previous studies conducted in France ${ }^{[29]}$. A better outcome is attributed to the shortened door-to-needle time created by more experienced neurologists ${ }^{[15]}$. A previous study showed that the mobile thrombolysis team with more experienced physicians but not personnel could shorten door-to-needle time ${ }^{[30]}$. In China, although there are clinical guidelines and stroke protocols, the fear of the risk of complications of thrombolysis, education level, and working years constitute the factors contributing to the in-hospital delay and low rate of thrombolysis for AIS $^{[31]}$.

In addition, since the big five personality traits were important for thrombolysis therapy for acute stroke among Chinese neurologists in this study, the big five personality traits should be applied to post-graduation education, such as standardized training which has been validated in other countries ${ }^{[32]}$, for neurologists residents, which also will meet the new medicine curriculum requirements ${ }^{[33]}$ in 
China. It might be necessary to provide individualized education program for resident during the post-graduation education according to their personality traits, and more subsequent studies are recommended to investigate how the unique constellation of personality traits impinge upon their medical practice, promote positive physician-patient interaction and relationship.

\section{Limitations}

Our study has several limitations. Firstly, the study hospital is located in East China, with a relatively economically stable population compared with Midwest China; thereby, generalizing our findings maybe is not practical. Secondly, we did not include the data of younger physicians because they have only a few treatment experiences, which may cause bias. Finally, this is only a single-center study; hence, multicenter studies are required to assess the personality factors for the in-hospital delay, so that we can improve the care management for stroke patients.

\section{Conclusions}

Here, we found that the personality traits among physicians is associated with door-to-needle time for thrombolytic therapy in acute ischemic stroke. We demonstrated that extraversion trait shortened the in-hospital delay. However, the conscientiousness trait was shown to postpone the procedure of thrombolysis treatment. Moreover, the accumulation of the acquired clinical experience was shown to weaken the personality disadvantages in the thrombolytic therapy for acute ischemic stroke. Therefore, we recommend that the big five personality traits among physicians should be applied in post-graduation education.

\section{Abbreviations}

AIS: acute ischemic stroke; IVT: intravenous thrombolysis; DNT: door-to-needle time; ED: emergency department; CBF-PI-B: Chinese Big Five Personality Inventory Brief Version; SD: Sd. Deviation; OR: odds ratio; Cl:confidence interval; IQR: interquartile range; NIHSS: National Institutes of Health Stroke Scale; ODT: onset-to-door time; DTI: door-to-imaging time; DTL:door-to-laboratory time; FTN: finaltest-to-needle time

\section{Declarations}

\section{Acknowledgments}

We would like to thank Dr. Shenqiang Yan, Dr. Wenhua Zhang for their valuable help. We thank all of colleagues in emergency department for their collaboration.

\section{Authors' contributions}

Z Lu, Q Xia and S Xia designed the study. X Yang, H Ma and S Shen collected the data. Z Lu and Q Xia analyzed the data, Z Lu and Q Xia drafted the manuscript.

S Xia final approval of the manuscript.

\section{Funding}

This study was supported by grants from Zhejiang Province Public Welfare Technology Application Research Project (grant number: 2018RC070) and Huzhou Municipal Science and Technology Bureau (grant number: 2019GY05)

\section{Availability of data and materials}

Anonymised data analysed for the current study will be shared if a reasonable request is made by a qualified investigator to the corresponding author.

\section{Ethics approval and consent to participate}

The Human Subjects Review Committee of the Huzhou Central Hospital approved this research. Since only observational data were collected, written informed consent was not required according to law. 


\section{Consent for publication}

Not applicable.

\section{Competing interests}

The authors declare that they have no competing interests.

\section{Consent for publication}

Not applicable.

\section{References}

1. Dong Q, Dong Y, Liu L, Xu A, Zhang Y, Zheng H, et al. The Chinese Stroke Association scientific statement: intravenous thrombolysis in acute ischaemic stroke. Stroke Vasc Neurol. 2017;2:147-59. doi:10.1136/svn-2017-000074.

2. Powers WJ, Rabinstein AA, Ackerson T, Adeoye OM, Bambakidis NC, Becker K, et al. Guidelines for the Early Management of Patients With Acute Ischemic Stroke: 2019 Update to the 2018 Guidelines for the Early Management of Acute Ischemic Stroke: A Guideline for Healthcare Professionals From the American Heart Association/American Stroke Association. Stroke. 2019;50:e344-418. doi:10.1161/STR.0000000000000211.

3. Man S, Xian Y, Holmes DN, Matsouaka RA, Saver JL, Smith EE, et al. Association Between Thrombolytic Door-to-Needle Time and 1-Year Mortality and Readmission in Patients With Acute Ischemic Stroke. JAMA. 2020;323:2170-84. doi:10.1001/jama.2020.5697.

4. Kuhrij LS, Marang-van de Mheen PJ, van den Berg-Vos RM, de Leeuw FE, Nederkoorn PJ. Determinants of extended door-toneedle time in acute ischemic stroke and its influence on in-hospital mortality: results of a nationwide Dutch clinical audit. BMC Neurol. 2019;19:265. doi:10.1186/s12883-019-1512-2.

5. Kamal N, Smith EE, Jeerakathil T, Hill MD. Thrombolysis. Improving door-to-needle times for ischemic stroke treatment - A narrative review. Int J Stroke. 2018;13:268-76. doi:10.1177/1747493017743060.

6. Ormseth CH, Sheth KN, Saver JL, Fonarow GC, Schwamm LH. The American Heart Association's Get With the Guidelines (GWTG)Stroke development and impact on stroke care. Stroke Vasc Neurol. 2017;2:94-105. doi:10.1136/svn-2017-000092.

7. Kernberg OF. What is Personality? J Pers Disord. 2016;30:145-56. DOI:10.1521/pedi.2106.30.2.145.

8. John OP, Naumann LP, Soto CJ. Paradigm shift to the integrative big five trait taxonomy: History, measurement, and conceptual issues// Handbook of personality: Theory and research (3rd edition). 2008.

9. Goldberg LR. An alternative "description of personality": the big-five factor structure. J Pers Soc Psychol. 1990;59:1216-29. doi:10.1037//0022-3514.59.6.1216.

10. Gosling SD, Rentfrow PJ. SwannWB. A very brief measure of the Big-Five personality domains. J Res Pers. 2003;37:504-28. doi:10.1016/S0092-6566(03)00046-1.

11. Surbeck W, Samuel R, Spieler D, Seifritz E, Scantamburlo G, Stienen MN, et al. Neurologists, neurosurgeons, and psychiatrists' personality traits: a comparison. Acta Neurochir (Wien). 2020;162:461-8. doi:10.1007/s00701-020-04233-9.

12. Wang M-C, Dai X. Development of the Chinese Big Five Personality Inventory (CBF-PI): psychometric properties of CBF-PI brief version. Chinese Journal of Clinical Psychology. 2011;19:454-7. doi:10.1007/s10570-010-9464-0.

13. TARGET: STROKE PHASE II, Campaign Manual. 2014. Accessed August 2, 2016.http://www.strokeassociation.org/idc/groups/heartpublic/@wcm/@gwtg/documents/downloadable/ucm_470730.pdf.

14. Emberson J, Lees KR, Lyden P, Blackwell L, Albers G, Bluhmki E, et al. Effect of treatment delay, age, and stroke severity on the effects of intravenous thrombolysis with alteplase for acute ischaemic stroke: a meta-analysis of individual patient data from randomised trials. Lancet. 2014;384:1929-35. doi:10.1016/S0140-6736(14)60584-5.

15. Darehed D, Blom M, Glader EL, Niklasson J, Norrving B, Eriksson M. In-Hospital Delays in Stroke Thrombolysis: Every Minute Counts. Stroke. 2020;51(8):2536-9. doi:10.1161/STROKEAHA.120.029468.

16. Nepal G, Yadav JK, Basnet B, Shrestha TM, Kharel G, Ojha R. Status of prehospital delay and intravenous thrombolysis in the management of acute ischemic stroke in Nepal. BMC Neurol. 2019;19:155. doi:10.1186/s12883-019-1378-3. 
17. Heikkilä I, Kuusisto H, Stolberg A, Palomäki A. Stroke thrombolysis given by emergency physicians cuts in-hospital delays significantly immediately after implementing a new treatment protocol. Scand J Trauma Resusc Emerg Med. 2016;24:46. doi:10.1186/s13049-016-0237-0.

18. Wu S, Wu B, Liu M, Chen Z, Wang W, Anderson CS, et al. Stroke in China: advances and challenges in epidemiology, prevention, and management. Lancet Neurol. 2019;18:394-405. doi:10.1016/S1474-4422(18)30500-3.

19. Huang Q, Ma QF, Feng J, Cheng WY, Jia JP, Song HQ, et al. Factors Associated with In-Hospital Delay in Intravenous Thrombolysis for Acute Ischemic Stroke: Lessons from China. PLoS One. 2015;10:e0143145. doi:10.1371/journal.pone.0143145.

20. The Lancet. Protecting Chinese doctors. Lancet. 2020;395:90. doi:10.1016/S0140-6736(20)30003-9.

21. Pajonk FG, Andresen B, Schneider-Axmann T, Teichmann A, Gärtner U, Lubda J, et al. Personality traits of emergency physicians and paramedics. Emerg Med J. 2011;28:141-6. doi:10.1136/emj.2009.083311.

22. Tsoi W-F. Mental health in Singapore and its relation to Chinese culture. In: Tseng W-S, Wu Y-H, editors. Chinese culture and mental health. Orlando: Academic Press; 1985. pp. 229 - 250.

23. Hojat M, Gonnella JS, Nasca TJ, Mangione S, Vergare M, Magee M. Physician empathy: definition, components, measurement, and relationship to gender and specialty. Am J Psychiatry. 2002;159:1563-9. doi:10.1176/appi.ajp.159.9.1563.

24. Song Y, Shi M. Associations between empathy and big five personality traits among Chinese undergraduate medical students. PLoS One. 2017;12:e0171665. doi:10.1371/journal.pone.0171665.

25. Abe K, Niwa M, Fujisaki K, Suzuki Y. Associations between emotional intelligence, empathy and personality in Japanese medical students. BMC Med Educ. 2018;18:47. doi:10.1186/s12909-018-1165-7.

26. Smith MM, Sherry SB, Vidovic V, Saklofske DH, Stoeber J, Benoit A. Perfectionism and the Five-Factor Model of Personality: A Meta-Analytic Review. Pers Soc Psychol Rev. 2019;23:367-90. doi:10.1177/1088868318814973.

27. MacDougall EH, McCann S. The relation of neuroticism and social anxiety to willingness to volunteer. J Soc Psychol. 2020;160:459-64. doi:10.1080/00224545.2019.1677548.

28. Pérez-Fuentes M, Molero Jurado M, Martos M. Burnout and Engagement: Personality Profiles in Nursing Professionals. J Clin Med. 2019;8:286. doi:10.3390/jcm8030286.

29. Tuffal A, Moulin S, Dequatre-Ponchelle N, Bodenant M, Dumont F, Lefebvre C, et al. Influence of neurologists' experience on the outcome of patients treated by intravenous thrombolysis for cerebral ischaemia. J Neurol. 2015;262:1209-15. doi:10.1007/s00415-015-7693-8.

30. Hebant B, Triquenot-Bagan A, Guegan-Massardier E, Ozkul-Wermester O, Maltête D. In-hospital delays to stroke thrombolysis: Out of hours versus regular hours and reduction in treatment times through the creation of a 24/7 mobile thrombolysis team. J Neurol Sci. 2018;392:46-50. doi:10.1016/j.jns.2018.07.009.

31. Zhou Y, Yan S, Song X, Gong Y, Li W, Wang M, et al. Intravenous thrombolytic therapy for acute ischemic stroke in Hubei, China: a survey of thrombolysis rate and barriers. BMC Neurol. 2019;19:202. doi:10.1186/s12883-019-1418-z.

32. Moug SJ, Henderson N, Tiernan J, Bisset CN, Ferguson E, Harji D, et al. The colorectal surgeon's personality may influence the rectal anastomotic decision. Colorectal Dis. 2018;20:970-80. doi:10.1111/codi.14293.

33. Wang MD, Yin XX, Yang TT, Wang Y, Zhu YY, Zhou YF, et al. Chinese neurologists' perspective on intravenous thrombolysis for acute ischemic stroke. Brain Behav. 2018;8:e00882. doi:10.1002/brb3.882.

\section{Figures}




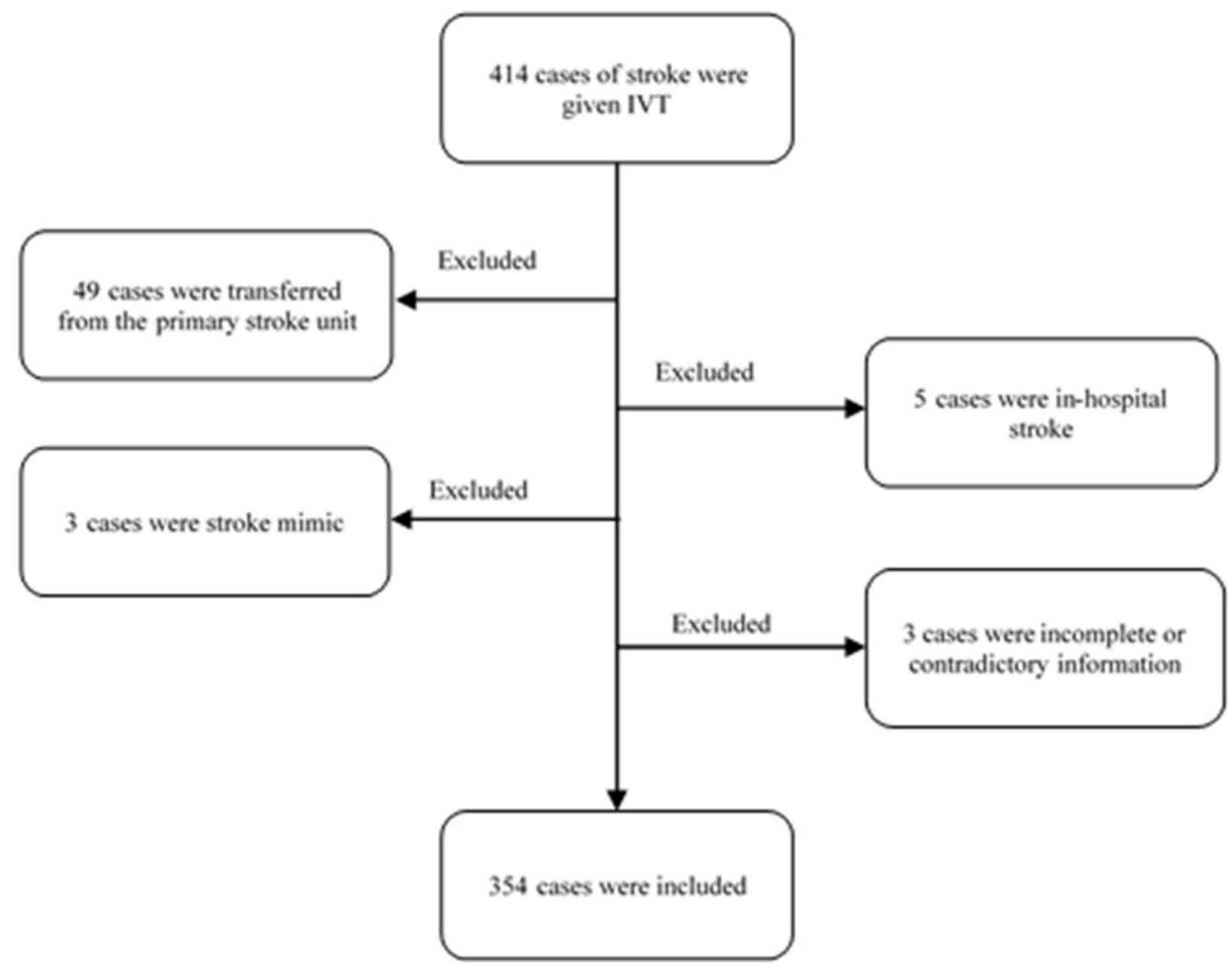

Figure 1

Flow diagram of patient selection 


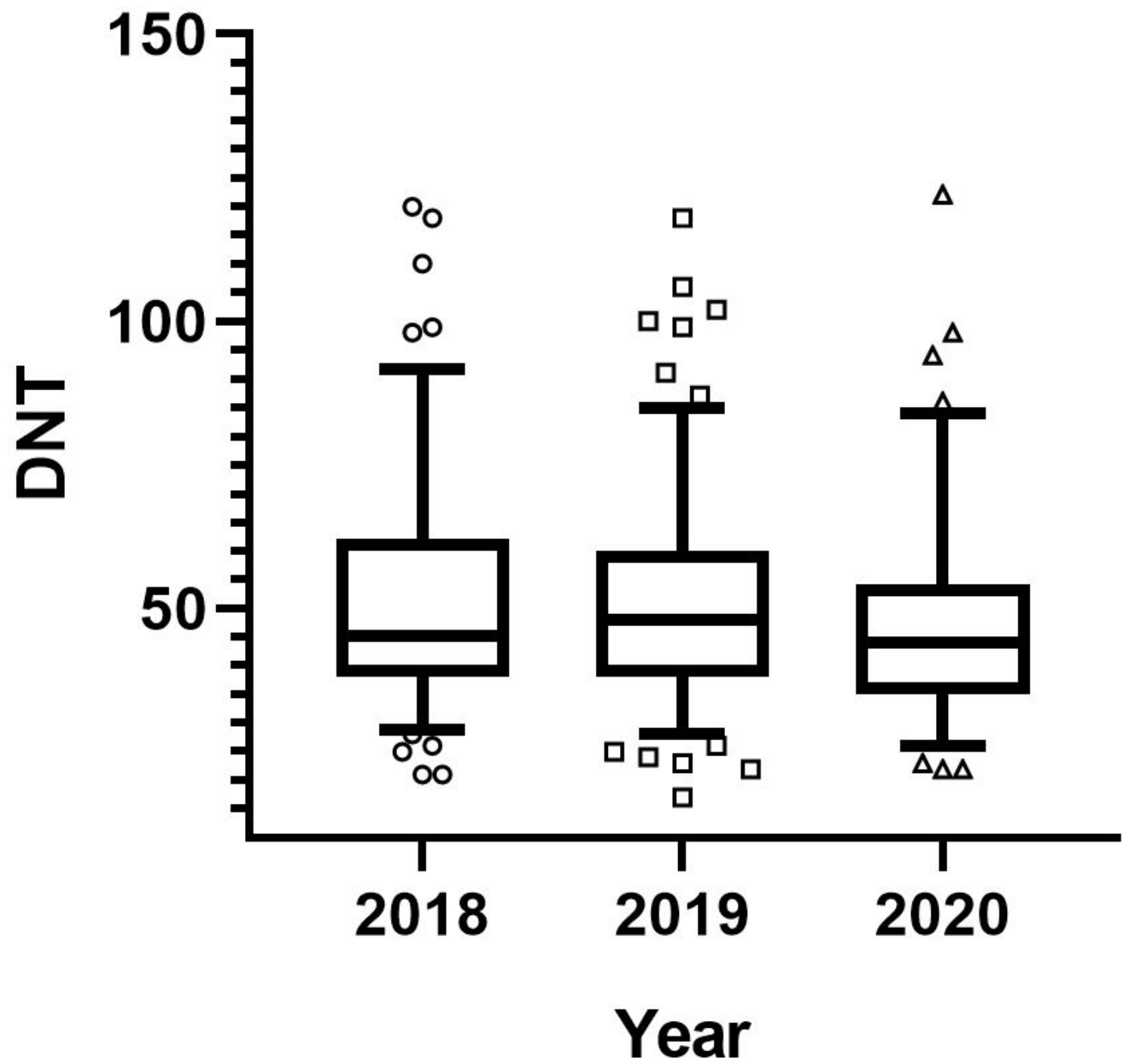

Figure 2

The median $(95 \% \mathrm{Cl})$ of DNT in three years DNT: door-to-needle times 


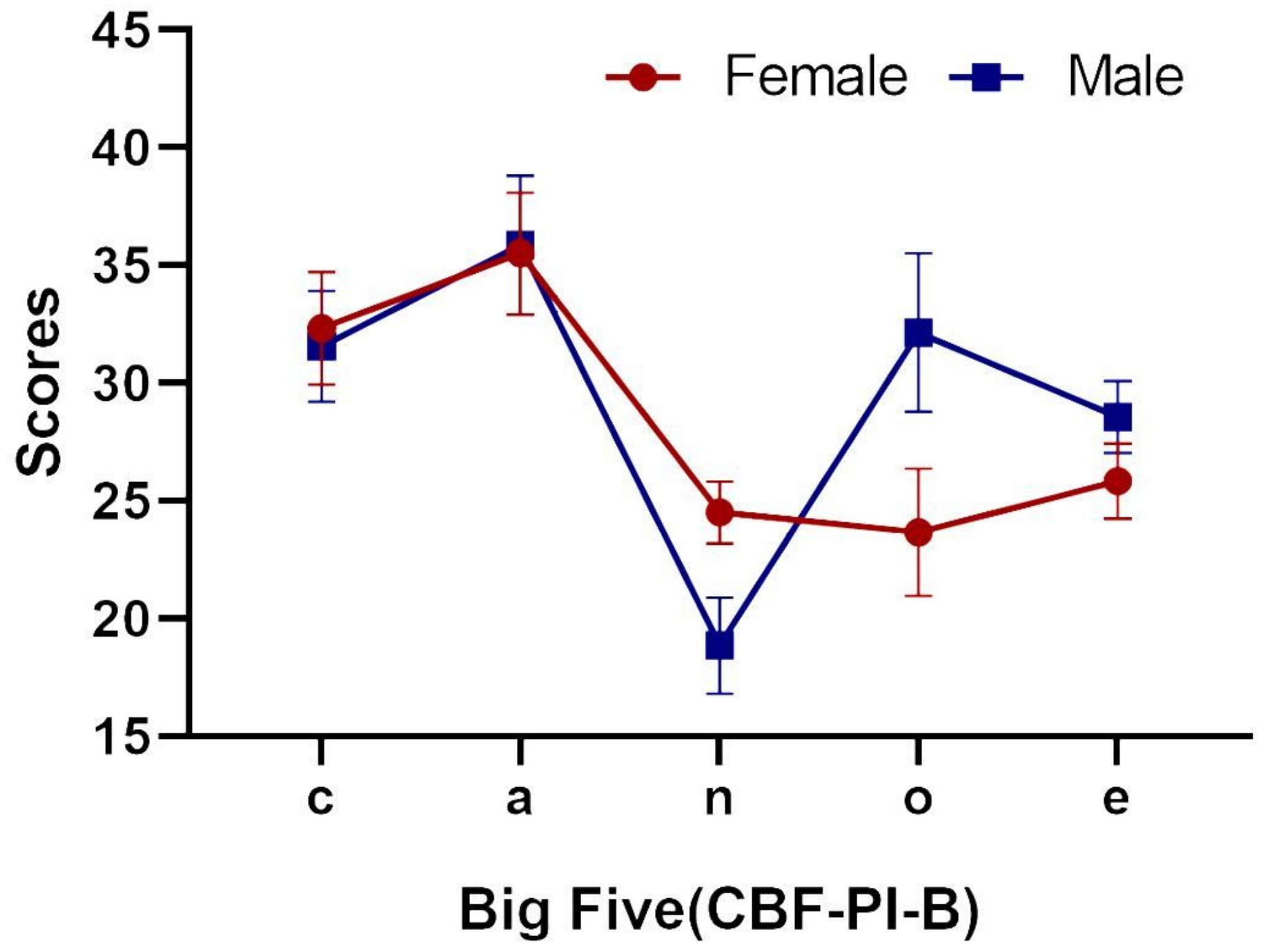

Figure 3

The mean scores and standard error for each of the Big Five factors of personality traits for gender differences,. CBF-PI-B: Chinese Big Five Personality Inventory Brief Version, C (conscientiousness), A (agreeableness), N (neuroticism), O (openness), E (extraversion) 
A
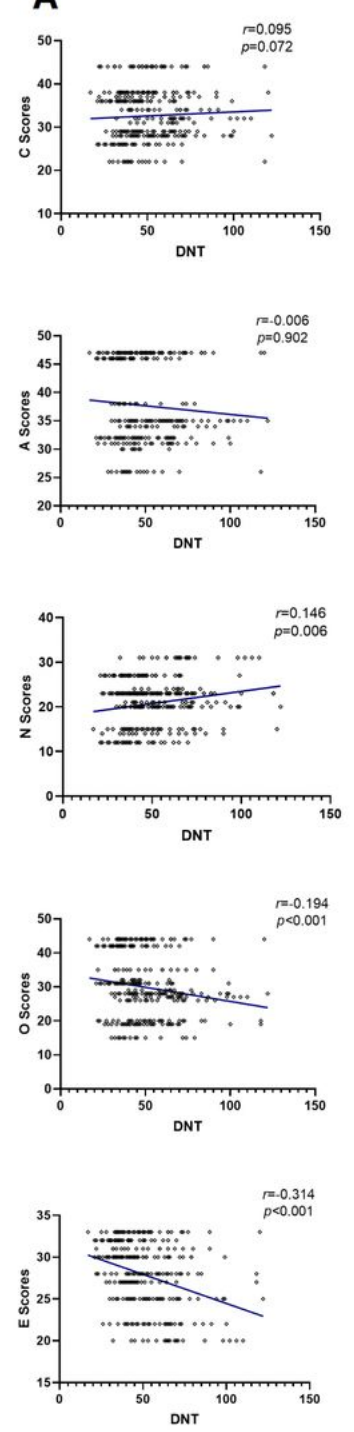

B
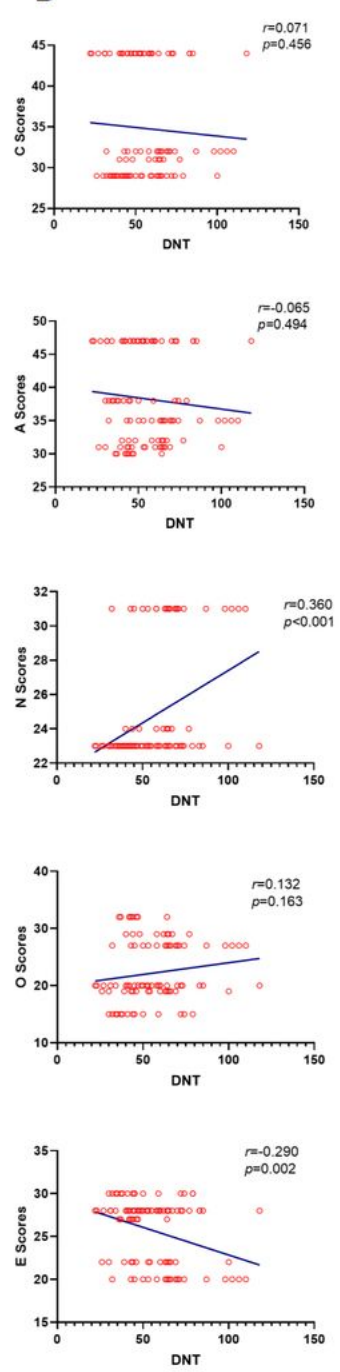
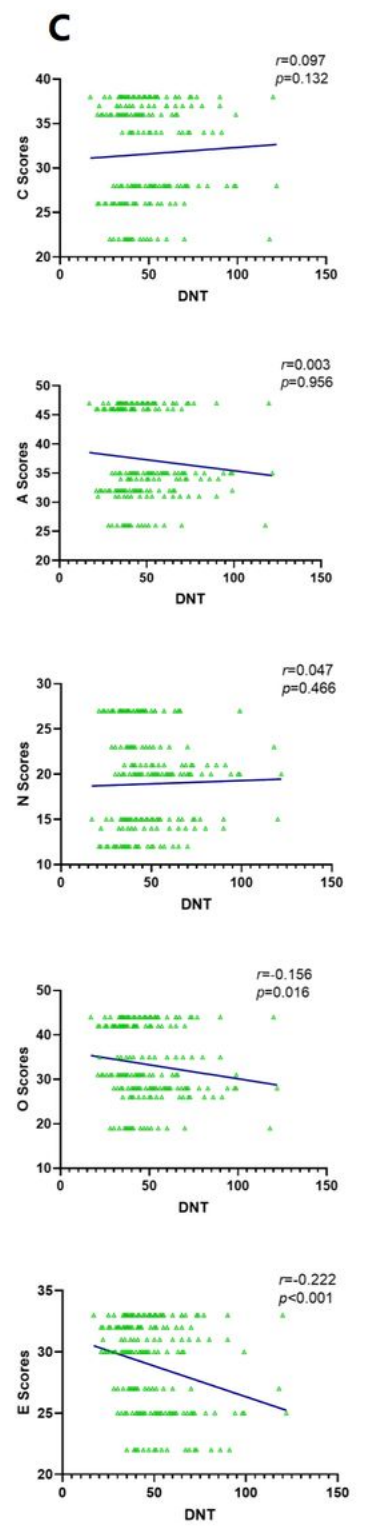

\section{Figure 4}

The relationship between the DNT and the scores of a-C (conscientiousness), b-A (agreeableness), c-N (neuroticism), d-O (openness), e-E (extraversion), among all physicians(A), female physicians(B) and male physicians(C). DNT: door-to-needle times 


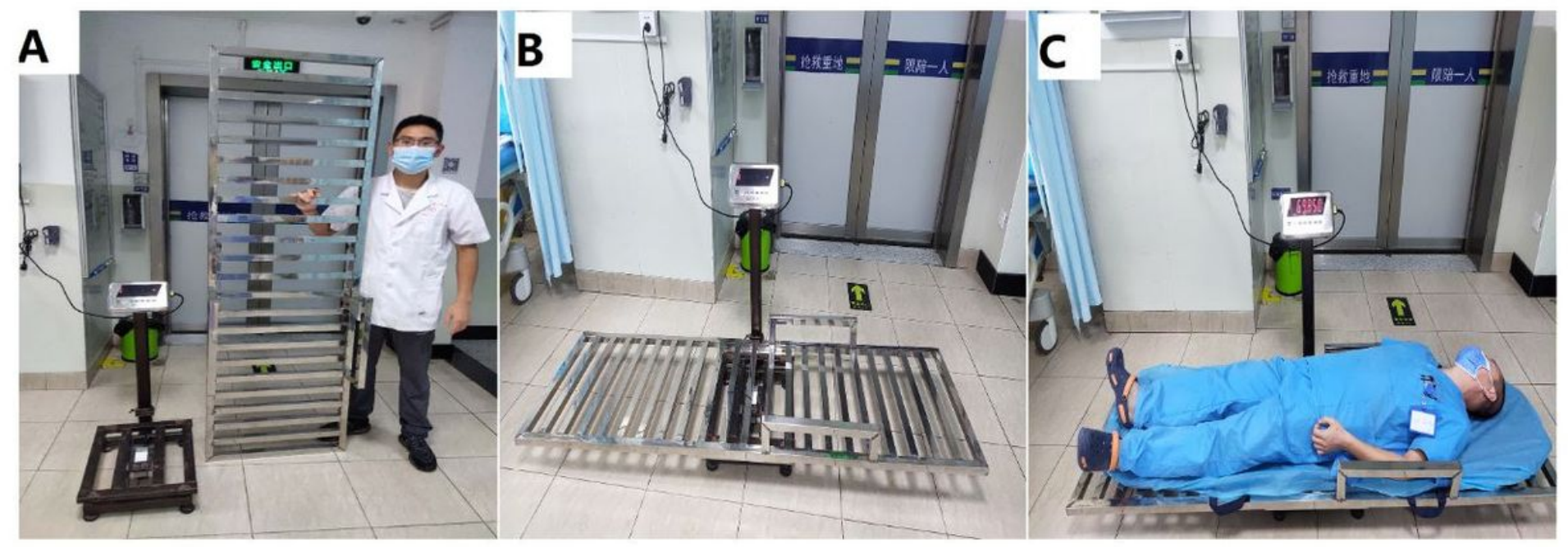

Figure 5

The self-made weighing scale, A: Preparation, B: Assembly, C: Weighing 